\title{
Cognitive and developmental outcomes after pediatric insular epilepsy surgery for focal cortical dysplasia
}

\author{
Naoki Ikegaya, MD,1,2 Masaki Iwasaki, MD, PhD,1 Yuu Kaneko, MD,, Takanobu Kaido, MD, PhD,,1,3 \\ Yuiko Kimura, MD, PhD, ${ }^{1}$ Tetsuya Yamamoto, MD, PhD, ${ }^{2}$ Noriko Sumitomo, MD, ${ }^{4}$ \\ Takashi Saito, MD, ${ }^{4}$ Eiji Nakagawa, MD, PhD, ${ }^{4}$ Kenji Sugai, MD, PhD, ${ }^{4}$ Masayuki Sasaki, MD, PhD, ${ }^{4}$ \\ Akio Takahashi, MD, PhD, ${ }^{1,5}$ and Taisuke Otsuki, MD, PhD',6 \\ Departments of ${ }^{1}$ Neurosurgery and ${ }^{4}$ Child Neurology, Epilepsy Center, National Center Hospital, National Center of Neurology \\ and Psychiatry (NCNP), Kodaira, Tokyo; ' ${ }^{2}$ epartment of Neurosurgery, Epilepsy Center, Yokohama City University School of \\ Medicine, Yokohama, Kanagawa; ' $D$ Department of Health and Nutrition, Faculty of Health and Nutrition, Osaka Shoin Women's \\ University, Higashi-Osaka, Osaka; ${ }^{5}$ Department of Neurosurgery, Shibukawa Medical Center, Shibukawa, Gunma; and ${ }^{6}$ Epilepsy \\ Hospital Bethel Japan, Iwanuma, Miyagi, Japan
}

OBJECTIVE Cognitive risk associated with insular cortex resection is not well understood. The authors reviewed cognitive and developmental outcomes in pediatric patients who underwent resection of the epileptogenic zone involving the insula.

METHODS A review was conducted of 15 patients who underwent resective epilepsy surgery involving the insular cortex for focal cortical dysplasia, with a minimum follow-up of 12 months. The median age at surgery was 5.6 years (range 0.3-13.6 years). Developmental/intelligence quotient (DQ/IQ) scores were evaluated before surgery, within 4 months after surgery, and at 12 months or more after surgery. Repeated measures multivariate ANOVA was used to evaluate the effects on outcomes of the within-subject factor (time) and between-subject factors (resection side, anterior insular resection, seizure control, and antiepileptic drug $[A E D]$ reduction).

RESULTS The mean preoperative $D Q / I Q$ score was $60.7 \pm 22.8$. Left-side resection and anterior insular resection were performed in 9 patients each. Favorable seizure control (International League Against Epilepsy class 1-3) was achieved in 8 patients. Postoperative motor deficits were observed in 9 patients (permanent in 6, transient in 3). Within-subject changes in $D Q / I Q$ were not significantly affected by insular resection $(p=0.13)$. Postoperative changes in $D Q / I Q$ were not significantly affected by surgical side, anterior insular resection, AED reduction, or seizure outcome. Only verbal function showed no significant changes before and after surgery and no significant effects of within-subject factors.

CONCLUSIONS Resection involving the insula in children with impaired development or intelligence can be performed without significant reduction in $\mathrm{DQ} / \mathrm{IQ}$, but carries the risk of postoperative motor deficits.

https://thejns.org/doi/abs/10.3171/2020.5.PEDS2058

KEYWORDS epilepsy surgery; insula; focal cortical dysplasia; cognitive; developmental

$\mathrm{R}$ ESECTION of the insula for the treatment of epilepsy is technically challenging, but achieves satisfactory amelioration. ${ }^{1-9}$ However, the neurological and neuropsychological sequelae remain unclear compared with other epilepsy surgeries, because little evidence for postoperative functional outcomes has been reported. Knowledge of the possible neurological sequelae is crucial in the selection of the most appropriate treatment strategy. The risk of decreases in motor function is increasingly understood, ${ }^{4,10}$ but the risk of cognitive dysfunction is still under investigation.

Direct cortical stimulation (DCS) and functional neuroimaging studies have revealed the varied and complex functions of the insula, including both visceral and sen-

ABBREVIATIONS AED = antiepileptic drug; CST = corticospinal tract; $D C S=$ direct cortical stimulation; $D Q=$ developmental quotient; FCD = focal cortical dysplasia; IED = interictal epileptic discharge; ILAE = International League Against Epilepsy; IQ = intelligence quotient; KIDS = Kinder Infant Development Scale; MEG = magnetoencephalography; SISCOM = subtraction ictal SPECT coregistered to MRI; T-B test = Tanaka-Binet test; WISC = Wechsler Intelligence Scale for Children.

SUBMITTED January 23, 2020. ACCEPTED May 7, 2020.

INCLUDE WHEN CITING Published online August 7, 2020; DOI: 10.3171/2020.5.PEDS2058. 
sory functions..$^{11,12}$ In addition, the insula is related to various motor, auditory, vestibular, and autonomic functions; emotion and behavior; and cognitive functions such as attention, working memory, speech, and language. ${ }^{11-14}$ These different functions are organized in distinct regions inside the insula. The anterior insula, which is connected to the frontal region, is most involved in cognitive function. ${ }^{11}$ Furthermore, the left insula is more active and responsive to speech and language tasks. ${ }^{15}$ Thus, resection of the anterior and left insula may have important effects on postoperative cognitive function.

DCS and functional neuroimaging monitors spontaneous or task-evoked functional activation but does not always predict the dysfunctions caused by resection. However, clinical evidence shows that damage to the insula is related to cognitive dysfunction, including language function. ${ }^{16}$ Therefore, the possibility that insular resection results in cognitive changes must be clarified.

Knowledge of the impact of insular epilepsy surgery on cognitive function is limited. Evaluation with multiple neuropsychological batteries before and after surgery found that mild impairment of oromotor speed can occur despite the absence of major neuropsychological effects. ${ }^{17}$ However, postoperative cognitive function was also found to remain unchanged or was improved..$^{2,18}$

Cognitive function disturbance in pediatric epilepsy is often related to the underlying etiology, seizures, age at onset, and potentially epileptiform abnormalities and antiepileptic drugs (AEDs). ${ }^{19}$ The potential for amelioration of cognitive function subsequent to control of epilepsy is one of the most important motivations for surgery. Focal cortical dysplasia (FCD) is a major etiology in pediatric epilepsy surgery. FCD is structurally pathological but may affect physiological functions. ${ }^{20}$ Therefore, functional gain achieved by epilepsy control must be balanced against the functional loss caused by resection. Consequently, the potential for such changes must be carefully evaluated and considered before deciding on a surgical strategy.

The present study evaluated the risk to cognitive functions of insular surgery by comparing neuropsychological and developmental assessments, including cognitive function, before and after surgery for FCD.

\section{Methods \\ Patients}

We retrospectively reviewed patients with drug-resistant epilepsy caused by FCD who underwent resection of the epileptogenic zone involving the insula between October 2006 and February 2016 in the National Center Hospital, National Center of Neurology and Psychiatry. Other criteria included follow-up after epilepsy surgery for at least 12 months and availability of regular developmental and neuropsychological assessments. Patients who had previously undergone epilepsy surgery in the extrainsular region were included, whereas patients who underwent extensive multilobe resection or disconnection, or hemispherotomy, were excluded. Medical charts were reviewed to determine the patient characteristics, including age at surgery, sex, handedness, duration of epilepsy, and AEDs. The ethics committee of the IRB approved this study, and written informed consent was obtained from all parents and/or guardians.

\section{Pre- and Postoperative Evaluations}

Our routine presurgical evaluation includes long-term video EEG monitoring, 3-T MRI, FDG-PET, subtraction ictal SPECT coregistered to MRI (SISCOM), and magnetoencephalography (MEG). The extent of abnormal findings in the insula was divided into two groups: an abnormal area located in the short insular gyrus was defined as an anterior insula lesion, and an abnormal area located in the long insular gyrus was defined as a posterior insula lesion. The optimal surgical approach was determined after detailed discussions at a multidisciplinary epilepsy surgery conference. Epileptic seizures recorded by videoEEG monitoring were classified and confirmed at this conference. Seizure types were reclassified according to the International League Against Epilepsy (ILAE) 2017 classification. ${ }^{21}$

Neuropsychological assessment was based on intelligence quotient (IQ) determined with the Wechsler Intelligence Scale for Children (WISC) III or IV, or the Tanaka-Binet test (T-B test), which is the standardized Japanese version of the Stanford-Binet test. However, patients younger than the age limit for the IQ test or who were too intellectually impaired for IQ testing were assessed with the developmental quotient (DQ) using the Kinder Infant Development Scale (KIDS). This caregiver-rated DQ scale consists of 6 components, including gross motor movement, fine motor movement, language comprehension, language expression, social relationships, and feeding. ${ }^{22}$ The index is calculated as the percentage ratio of the developmental age to the calendar age. This index was adopted in the same way as IQ in a previous study. ${ }^{23}$ IQ and DQ were evaluated before surgery, within 4 months after surgery (first assessment), and at 12 months or more after surgery (last assessment).

\section{Surgery}

All patients underwent the subpial transopercular approach to the insula, and no patient underwent pure insulectomy. Corticectomy of the lobe surrounding the insula was usually combined with insulectomy. Drastic surgical intervention primarily aimed at seizure control and/or developmental improvement was occasionally performed in exchange for neurological deficits such as motor paralysis. The insular cortex was removed using bipolar cauterization and suction and/or ultrasonic aspiration. The white matter underneath the insular cortex (extreme capsule) was set as the limit of the bottom of resection, and the procedure was performed meticulously to avoid direct injury to the basal ganglia and lenticulostriate artery. A specimen of the insular cortex was extracted if safely possible during removal. The extent of resection was assessed on postoperative CT and MRI.

\section{Statistical Analysis}

Evaluation of the effect of insula resection on IQ and DQ used repeated measures, multivariate ANOVA with the preoperative index, first postoperative index, and last 
TABLE 1. Patient characteristics and seizures

\begin{tabular}{|c|c|c|c|c|c|c|}
\hline \multirow{2}{*}{$\begin{array}{l}\text { Case No. } \\
\text { (Sex) }\end{array}$} & \multicolumn{2}{|c|}{ Age } & \multirow{2}{*}{$\begin{array}{l}\text { Side of } \\
\text { Surgery }\end{array}$} & \multirow[b]{2}{*}{ Handedness } & \multicolumn{2}{|r|}{ Seizure } \\
\hline & Onset & Surgery & & & Type & Semiology \\
\hline $1(\mathrm{~F})$ & 4 mos & 2 yrs 5 mos & $\mathrm{Lt}$ & $\mathrm{Rt}$ & FIAS & $\begin{array}{l}\text { Eye-blinking, eye-deviation to rt, laryngeal constriction/breath-holding w/ } \\
\text { impaired awareness }\end{array}$ \\
\hline $2(\mathrm{~F})$ & $1 \mathrm{yr}$ & 4 yrs 9 mos & Rt & Rt & FMS & $\begin{array}{l}\text { Eye-blinking, asymmetrical tonic posturing (It arm extension, rt arm } \\
\text { extension \& elevation) }\end{array}$ \\
\hline $3(\mathrm{~F})$ & $11 \mathrm{mos}$ & 6 yrs 8 mos & Rt & Rt & FMS, FIAS & Lt arm \& face tonic seizure, hyperkinetic behavior w/ impaired awareness \\
\hline $4(F)$ & $1 \mathrm{mo}$ & 3 yrs 8 mos & Rt & Unknown & FIAS & Behavior arrest $w /$ impaired awareness \\
\hline $5(\mathrm{~F})$ & $1 \mathrm{mo}$ & 13 yrs 7 mos & $\mathrm{Lt}$ & $\mathrm{Rt}$ & FMS & Asymmetrical tonic seizure (rt arm extension, It arm flexion) \\
\hline $6(\mathrm{~F})$ & 4 mos & 5 yrs 7 mos & Rt & $\mathrm{Rt}$ & FMS & Lt arm tonic seizure triggered by surprise stimulus \\
\hline $7(\mathrm{M})$ & 3 yrs 4 mos & 7 yrs 5 mos & $\mathrm{Lt}$ & Rt & FIAS & Behavior arrest w/ impaired awareness \\
\hline $8(\mathrm{M})$ & 3 yrs & 10 yrs $1 \mathrm{mo}$ & Lt & $\mathrm{Lt}$ & FIAS & Hyperkinetic behavior w/ impaired awareness \\
\hline $9(\mathrm{~F})$ & 6 yrs 5 mos & 8 yrs 3 mos & $\mathrm{Lt}$ & $\mathrm{Lt}$ & FSS, FMS & $\begin{array}{l}\text { Rt upper hand/rt side of the body numbness, rt upper limb tonic seizure, } \\
\text { speech disturbance }\end{array}$ \\
\hline $10(\mathrm{~F})$ & $2 \mathrm{mos}$ & $5 \mathrm{mos}$ & $\mathrm{Rt}$ & Unknown & FMS & Asymmetrical tonic posturing (rt arm extension, It arm elevation) \\
\hline $11(\mathrm{M})$ & 9 mos & 12 yrs 5 mos & Lt & $\mathrm{Rt}$ & FMS, FIAS & $\begin{array}{l}\text { Rt arm tonic seizure, symmetrical tonic posturing (rt arm extension) w/ } \\
\text { impaired awareness }\end{array}$ \\
\hline $12(\mathrm{M})$ & 4 mos & $1 \mathrm{yrs} 9 \mathrm{mos}$ & $\mathrm{Lt}$ & Unknown & FIAS, FMS & $\begin{array}{l}\text { Eye-blinking, breath-holding w/ impaired awareness, asymmetrical tonic } \\
\text { posturing (rt arm extension, It arm elevation) }\end{array}$ \\
\hline $13(F)$ & $7 \mathrm{mos}$ & 2 yrs 10 mos & $\mathrm{Rt}$ & $\mathrm{Rt}$ & FMS & $\begin{array}{l}\text { Vocalization, asymmetrical tonic posturing (It arm extension, rt arm } \\
\text { elevation) }\end{array}$ \\
\hline $14(\mathrm{~F})$ & $4 \mathrm{mos}$ & 13 yrs 3 mos & $\mathrm{Lt}$ & Rt & FIAS & Behavior arrest w/ impaired awareness \\
\hline $15(\mathrm{M})$ & $1 \mathrm{mo}$ & 4 mos & Lt & Unknown & FMS, FES & Rt face clonic seizure, asymmetrical spasm \\
\hline
\end{tabular}

FES = focal epileptic spasm; FIAS = focal impaired awareness seizure; FMS = focal motor seizure; FSS = focal sensory seizure.

postoperative index as within-subject factors (time). Surgical side (left/right), anterior insular resection (yes/no), seizure outcome (good, ILAE classes 1-3; poor, classes 4-6), and AED reduction after surgery were used as between- subject factors. Anterior insular resection was used as a between-subject factor because the functional topography of neuroimaging experiments suggests that the anterior insula is related to cognitive task processing..$^{11}$ To exclude

TABLE 2. EEG, MEG, and imaging findings

\begin{tabular}{|c|c|c|c|c|c|c|}
\hline \multirow{2}{*}{$\begin{array}{c}\text { Case } \\
\text { No. }\end{array}$} & \multicolumn{2}{|r|}{ Scalp EEG } & \multirow[b]{2}{*}{ MEG } & \multicolumn{3}{|c|}{ Imaging Findings at the Insula } \\
\hline & Location of IEDs & Location of Ictal EEG Onset & & MRI Lesion & FDG-PET & SISCOM* \\
\hline 1 & Lt mT, pT, P & Lt mT, pT, P & No spikes & Post & Hypometabolism & Yes \\
\hline 2 & Rt C, pT, midline C & Rt C, F, P & Rt T-op cluster & Ant \& Post & Hypometabolism & Yes \\
\hline 3 & Rt F, C, aT & Rt C, F, Fp, mT & Rt F, P, T scatter & Ant \& Post & Hypometabolism & Yes \\
\hline 4 & Rt Fp, F, aT & Rt Fp, F, Fz & Rt F scatter & Negative & Hypometabolism & Yes \\
\hline 5 & $\mathrm{Lt} F, \mathrm{C}, \mathrm{P}$ & LtF & Lt F cluster & Negative & NA & Yes \\
\hline 6 & Rt P, F, C, pT & Rt C, F, P & Rt P-op cluster & Ant \& Post & Hypometabolism & No \\
\hline 7 & Lt Fp, F, aT & NA & Lt F cluster & Negative & NA & Yes \\
\hline 8 & Lt aT, mT, pT, C, P & Lt aT, mT, pT, P & Lt F cluster & Negative & Hypometabolism & No \\
\hline 9 & Lt mT, pT, C, P & Lt C, P & Lt F-op, P-op cluster & Negative & NA & Yes \\
\hline 10 & Rt F, C, P & Rt P, mT & Rt F, C, F-op cluster & Ant \& Post & Hypermetabolism & No \\
\hline 11 & Diffuse & Rt F, Fp, aT & Lt P-op cluster & Ant & Hypometabolism & No \\
\hline 12 & Lt mT, pT & Lt mT, pT & Lt T-op, P-op cluster & Post & NA & Yes \\
\hline 13 & Diffuse & Rt F & Bilat F scatter & Ant & Hypometabolism & Yes \\
\hline 14 & Lt aT, mT & Lt aT, mT, pT & Lt T cluster & Negative & Hypometabolism & No \\
\hline 15 & Lt P, C & Lt P, C, pT & Lt P-op, P cluster & Post & Hypermetabolism & Yes \\
\hline
\end{tabular}

Ant $=$ anterior; $\mathrm{aT}=$ anterior temporal; $\mathrm{C}=$ central; $\mathrm{F}=$ frontal; $\mathrm{Fp}=$ frontal pole; $\mathrm{mT}=$ middle temporal; $\mathrm{NA}=$ not available; $\mathrm{op}=$ operculum; $\mathrm{P}=$ parietal; Post = posterior; $\mathrm{pT}=$ posterior temporal; $\mathrm{T}=$ temporal.

${ }^{*}$ Significant increase in blood flow. 
TABLE 3. Surgery and outcomes

\begin{tabular}{|c|c|c|c|c|c|c|c|c|}
\hline \multirow[b]{2}{*}{ Case No. } & \multirow{2}{*}{$\begin{array}{l}\text { Previous } \\
\text { Surgical Site }\end{array}$} & \multicolumn{2}{|c|}{ Extent of Resection } & \multirow[b]{2}{*}{ Postop Motor Deficit } & \multirow{2}{*}{$\begin{array}{c}\text { CST } \\
\text { Resection }\end{array}$} & \multirow{2}{*}{$\begin{array}{l}\text { Histology } \\
\text { (FCD type) }\end{array}$} & \multirow{2}{*}{$\begin{array}{l}\text { FU Period } \\
\text { (mos) }\end{array}$} & \multirow{2}{*}{$\begin{array}{l}\text { ILAE } \\
\text { Class }\end{array}$} \\
\hline & & Extra-Insula & Insula & & & & & \\
\hline \multicolumn{9}{|c|}{$\begin{array}{l}\text { Favorable sei- } \\
\text { zure outcome* }\end{array}$} \\
\hline 1 & $P$ & P-op, T-op & Post & No & & Ila & 22 & 1 \\
\hline 2 & $\mathrm{P}, \mathrm{T}$ & OFC, IFG, MFG, F-op & Ant \& Post & $\begin{array}{l}\text { Permanent (worsening of hemi- } \\
\text { paresis from mild-moderate to } \\
\text { moderate) }\end{array}$ & & Ila & 25 & 1 \\
\hline 3 & & P-op & Ant \& Post & $\begin{array}{l}\text { Transient (mild UE > LE hemiparesis } \\
\text { for } 3 \text { wks) }\end{array}$ & & Ila & 17 & 1 \\
\hline 4 & $\mathrm{~F}$ & MFG, F-op, T-op & Ant & $\begin{array}{l}\text { Transient (mild hemiparesis w/in } 2 \\
\text { wks) }\end{array}$ & & Ila & 27 & 1 \\
\hline 5 & $\mathrm{~F}$ & IFG, MFG, F-op & Ant & No & & Ilb & 24 & 1 \\
\hline 6 & & $\begin{array}{l}\text { IFG, MFG, F-op, PrCG, } \\
\text { P-op, PoCG, T-op }\end{array}$ & Ant \& Post & $\begin{array}{l}\text { Permanent (worsening of hemipare- } \\
\text { sis from mild to moderate) }\end{array}$ & Yes & Ic & 23 & 1 \\
\hline 7 & $\mathrm{~F}$ & $\begin{array}{l}\text { OFC, IFG, MFG, SFG, } \\
\text { F-op }\end{array}$ & Ant & No & & $\mathrm{lb}$ & 36 & 1 \\
\hline 8 & $\mathrm{~F}$ & MFG, F-op & Ant & No & & $\mathrm{lb}$ & 56 & 3 \\
\hline \multicolumn{9}{|l|}{$\begin{array}{l}\text { Poor seizure } \\
\text { outcome } \dagger\end{array}$} \\
\hline 9 & $P$ & F-op, P-op, T-op & Post & $\begin{array}{l}\text { Permanent (newly moderate hemi- } \\
\text { paresis) }\end{array}$ & Yes & Ila & 62 & 4 \\
\hline 10 & & OFC, IFG, MFG, F-op & Ant \& Post & $\begin{array}{l}\text { Permanent (newly moderate hemi- } \\
\text { paresis) }\end{array}$ & Yes & Ila & 35 & 4 \\
\hline 11 & & F-op, P-op & Post & Transient (mild UE paresis for 2 wks) & & $\mathrm{Ilb}$ & 70 & 5 \\
\hline 12 & $T$ & P-op, SMG, AG & Post & $\begin{array}{l}\text { Permanent (newly moderate hemi- } \\
\text { paresis) }\end{array}$ & & Ilb & 58 & 4 \\
\hline 13 & & $\begin{array}{l}\text { OFC, IFG, MFG, SFG, } \\
\text { F-op }\end{array}$ & Ant & No & & Ilb & 54 & 4 \\
\hline 14 & $\mathrm{~F}$ & F-op & Ant & No & & Ila & 45 & 4 \\
\hline 15 & & $\begin{array}{l}\text { P-op, PoCG, SPL, } \\
\text { SMG, T-op }\end{array}$ & Post & $\begin{array}{l}\text { Permanent (worsening of hemipare- } \\
\text { sis from mild to moderate) }\end{array}$ & Yes & Ic & 41 & 5 \\
\hline
\end{tabular}

$\mathrm{AG}=$ angular gyrus; FU = follow-up; IFG = inferior frontal gyrus; $\mathrm{LE}=$ lower extremities; $\mathrm{MFG}=$ middle frontal gyrus; $\mathrm{OFC}=$ orbitofrontal cortex; PoCG = postcentral gyrus; $\mathrm{PrCG}=$ precentral gyrus; $\mathrm{SFG}=$ superior frontal gyrus; $\mathrm{SMG}$ = supramarginal gyrus; SPL = superior parietal lobule; UE = upper extremities.

* ILAE classes $1-3$.

† ILAE classes 4-6.

the effect of motor complications, further analysis using the mean score of language comprehension and language expression in the DQ (KIDS) and verbal comprehension in the WISC was conducted as a verbal (nonmotor) index assessment. This examination of verbal index was implemented in all but 1 patient who was evaluated with the T-B test because an index for verbal abilities could not be extracted in this patient. Statistical analyses used JMP software (version 11, SAS Institute Inc.). A p value $<0.05$ was considered significant.

\section{Results}

Seventeen patients underwent resective epilepsy surgery involving the insula during the study period. However, 1 patient who underwent periinsular hemispherotomy 5 months after surgery and 1 patient whose developmental and neuropsychological assessments could not be followed up were excluded. Thus, this study included 15 patients (10 girls and 5 boys). Table 1 shows the preoperative clinical characteristics. All patients were younger than 15 years old (median 5.6 years, range $0.3-13.6$ years). Seizures began in 11 patients when they were less than 1 year old (median 0.3 years, range $0.1-6.5$ years). Various ictal symptoms were observed in these patients. However, the subjective ictal manifestations could not be fully described by these infants. All patients had daily seizures.

Table 2 shows the electrophysiological and imaging findings. Interictal epileptic discharges (IEDs) were widely or multiregionally distributed. Ictal recordings were obtained in all but 1 patient with behavioral problems, which prevented continued long-term recording. No patient showed IEDs or onset of ictal discharge in the occipital area. Preoperative MRI revealed increased cortical thickness and/or blurring of the gray-white matter junction of the insula in 9 patients (60\%). FDG-PET detected abnormal glucose metabolism in the insula in 11 patients 

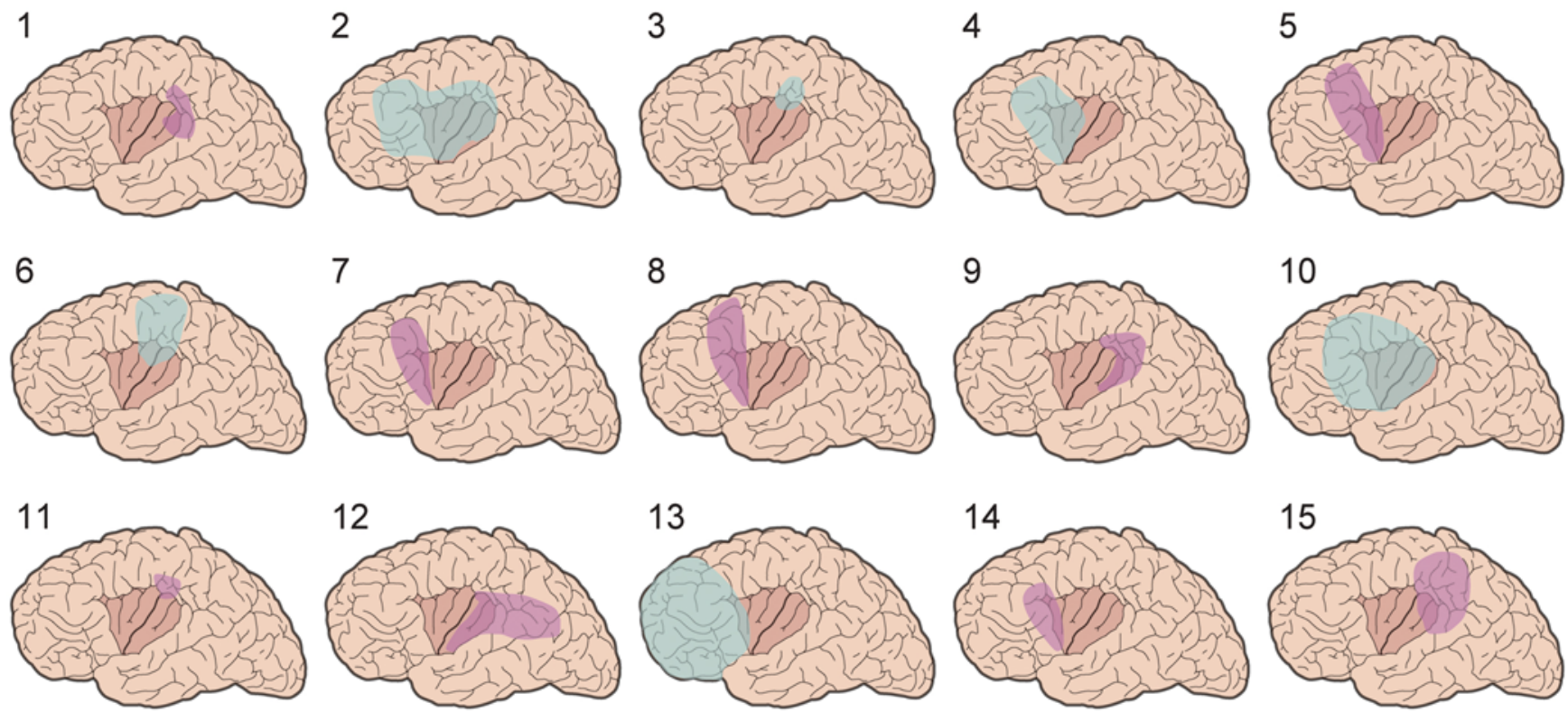

FIG. 1. Schematic representation of the extent of resection in each patient. Right-side resection (b/ue) is inverted to the same view as left-side resection (magenta). No patient underwent pure insulectomy. Opercular and extraopercular (lobar) resections were combined with insular corticectomy. Figure is available in color online only.

(73\%), including increased uptakes in 2 infants younger than 5 months. SISCOM was useful in more than half of the cases without MRI abnormalities.

Table 3 shows the type of surgery and outcomes. Favorable seizure outcomes were achieved in $8(53 \%)$ of 15 patients. Nine patients had undergone previous epilepsy surgery in the extrainsular region. Eight of 9 patients who underwent posterior insular resection developed postoperative motor complications (transient in 2 patients, permanent in 6 patients). In contrast, only 1 of 6 patients who underwent anterior insular resection developed transient paralysis. The 4 patients who developed permanent motor deficits underwent intentional removal of lesions overlapping the corticospinal tract (CST), giving priority to seizure control. All 15 patients underwent opercular resection in the surgical corridor. Ten patients also underwent further removal of the extraopercular cortex. The extent of resection is schematically presented for each patient in Fig. 1. Histopathological examination revealed FCD in all patients (type $\mathrm{I}, \mathrm{n}=4$; type $\mathrm{II}, \mathrm{n}=11$ ), although a specimen from the insula was obtained only in 8 patients. Specimens were sampled from the extrainsular region in the other 7 patients (operculum in 3, both operculum and frontal lobe in 4).

Table 4 shows the neuropsychological and developmental assessments and pre- and postoperative AEDs. Nine patients were tested for DQ and 6 were tested for IQ (WISC-III in 3, WISC-IV in 2, T-B test in 1). The mean period between the day of surgery and first postoperative assessment was 2.0 months (range $0.5-4.0$ months), and between the day of surgery and last postoperative assessment was 38.8 months (range 14.0-70.0 months). Developmental stagnation or regression was suspected preoperatively in 4 patients. The preoperative, first, and last postoperative $\mathrm{DQ} / \mathrm{IQ}$ indices $($ mean $\pm \mathrm{SD}$ ) were 60.7 $\pm 22.8,60.0 \pm 20.0$, and $56.0 \pm 20.9$, respectively (Fig. 2). Within-subject changes in DQ/IQ were not significantly affected by resection involving the insula $(\mathrm{p}=0.13$, Fig. $2 \mathrm{~A}$ ), or by the surgical side or anterior insular resection ( $\mathrm{p}$ $=0.43$ and 0.68 , respectively, Fig. $2 \mathrm{~B}$ and C). AED use was reduced in 8 patients, but was not associated with postoperative gain in DQ/IQ ( $p=0.83$, Fig. $2 \mathrm{D})$. The effect of seizure outcome did not reach significance $(p=0.18$, Fig. 2E). Evaluation limited only to the verbal index did not reach significance ( $p=0.07$, Fig. 3A). Between-subject factors also did not significantly affect the verbal index $(\mathrm{p}=0.64,0.86,0.49$, and 0.24 for resection side, anterior insular resection, AED reduction, and seizure outcome, respectively, Fig. 3B-E). None of the patients achieved improvement in verbal index of $10 \%$ or more.

\section{Discussion}

This study investigated the risk of cognitive dysfunction caused by epilepsy surgery involving resection of the insula by comparing pre- and postoperative IQ/DQ indices in 15 patients. In our series, epilepsy surgery involving the insula did not significantly affect cognitive or developmental function, although resection was not limited to the insula. Improvement in neuropsychological testing has been previously reported, ${ }^{2,18}$ but this study found no significant improvement. However, this series included many severe cases with early seizure onset and developmental regression, so preservation of function might be a positive effect of surgical treatment. Surgery in this area also carries the risk of postoperative motor paralysis. . $^{410}$

The insula is now considered important in the occurrence of focal epilepsy based on greater understanding of the pathology, clinical features, and effects of surgery, but the cognitive risk has been inadequately investigated. 
TABLE 4. Neuropsychological and developmental assessment and AEDs

\begin{tabular}{|c|c|c|c|c|c|c|c|c|c|c|c|}
\hline \multirow[b]{2}{*}{$\begin{array}{l}\text { Case } \\
\text { No. }\end{array}$} & \multirow[b]{2}{*}{$\begin{array}{l}\text { Preop Cognitive } \\
\text { Decline }\end{array}$} & \multirow[b]{2}{*}{$D Q / / Q$} & \multicolumn{3}{|c|}{ Global Index } & \multicolumn{3}{|c|}{ Verbal Index } & \multicolumn{2}{|c|}{ AED } & \multirow{2}{*}{$\begin{array}{c}\text { Decrease } \\
\text { in No. of } \\
\text { AEDs }\end{array}$} \\
\hline & & & Preop & $\begin{array}{l}\text { First } \\
\text { Postop }\end{array}$ & $\begin{array}{l}\text { Last } \\
\text { Postop }\end{array}$ & Preop & $\begin{array}{c}\text { First } \\
\text { Postop }\end{array}$ & $\begin{array}{l}\text { Last } \\
\text { Postop }\end{array}$ & Preop & Postop & \\
\hline \multicolumn{12}{|c|}{$\begin{array}{l}\text { Favorable sei- } \\
\text { zure outcome }\end{array}$} \\
\hline 1 & & $D Q$ & 100 & 86 & 87 & 107 & 107 & 118 & CLB, ZNS, KBr & CLB, ZNS & Yes \\
\hline 2 & & $\mathrm{DQ}$ & 83 & 82 & 85 & 93 & 85 & 82 & $\begin{array}{l}\text { ZNS, CLB, KBr, } \\
\text { RUF, PB }\end{array}$ & $\begin{array}{l}\text { ZNS, CLB, KBr, } \\
\text { RUF }\end{array}$ & Yes \\
\hline 3 & Yes (stagnation) & $\begin{array}{l}\text { IQ (WISC- } \\
\text { IV) }\end{array}$ & 67 & 57 & 55 & 68 & 69 & 68 & $\begin{array}{l}\text { CBZ, TPM, } \\
\text { KBr, GBP }\end{array}$ & CBZ, TPM, GBP & Yes \\
\hline 4 & & $\mathrm{DQ}$ & 59 & 51 & 53 & 54 & 49 & 52 & CBZ, CLB & CBZ, CLB & No \\
\hline 5 & & $\begin{array}{l}\text { IQ (WISC- } \\
\text { III) }\end{array}$ & 51 & 49 & 43 & 49 & 53 & 49 & $\begin{array}{l}\text { CBZ, PHT, } \\
\text { CLB, GBP }\end{array}$ & PHT, CLB, GBP & Yes \\
\hline 6 & & $\mathrm{DQ}$ & 43 & 47 & 49 & 57 & 56 & 54 & CBZ, TPM & $\mathrm{CBZ}$ & Yes \\
\hline 7 & & $D Q$ & 30 & 31 & 31 & 29 & 30 & 33 & ESM, CLB & CLB & Yes \\
\hline 8 & & $\mathrm{DQ}$ & 21 & 22 & 15 & 22 & 24 & 13 & CLB, ZNS & CLB, ZNS & No \\
\hline \multicolumn{12}{|c|}{$\begin{array}{l}\text { Poor seizure } \\
\text { outcome† }\end{array}$} \\
\hline 9 & Yes (regression) & $\begin{array}{l}\text { IQ (WISC- } \\
\text { III) }\end{array}$ & 96 & 93 & 78 & 106 & 97 & 84 & $\begin{array}{l}\text { PHT, CLB, LTG, } \\
\text { ZNS }\end{array}$ & $\begin{array}{l}\text { LTG, PHT, CLB, } \\
\text { LEV, RUF, DZP }\end{array}$ & No \\
\hline 10 & Yes (stagnation) & $\begin{array}{r}\mathrm{IQ}(\mathrm{T}-\mathrm{B} \\
\text { test })\end{array}$ & 88 & 85 & 83 & NA & NA & NA & $\begin{array}{l}\text { ZNS, TPM, PB, } \\
\quad \text { CLB }\end{array}$ & TPM, CLB, LTG & Yes \\
\hline 11 & & $\begin{array}{l}\text { IQ (WISC- } \\
\text { III) }\end{array}$ & 72 & 69 & 69 & 74 & 71 & 73 & $\begin{array}{l}\text { CBZ, TPM, } \\
\text { VPA, GBP }\end{array}$ & $\begin{array}{l}\text { CBZ, TPM, LEV, } \\
\text { CLB, PER }\end{array}$ & No \\
\hline 12 & & $\mathrm{DQ}$ & 57 & 48 & 35 & 42 & 38 & 34 & ZNS, PB & ZNS, PB, CBZ & No \\
\hline 13 & Yes (regression) & $\mathrm{DQ}$ & 55 & 65 & 41 & 61 & 66 & 51 & ZNS, VPA & ZNS, VPA, LEV & No \\
\hline 14 & & $\begin{array}{l}\text { IQ (WISC- } \\
\text { IV) }\end{array}$ & 50 & 48 & 49 & 55 & 51 & 54 & $\begin{array}{l}\text { CBZ, CLB, } \\
\text { TPM }\end{array}$ & $\begin{array}{l}\text { CBZ, CLB, CZP, } \\
\text { TPM }\end{array}$ & No \\
\hline 15 & & $\mathrm{DQ}$ & 38 & 67 & 67 & 67 & 67 & 68 & ZNS, CLB & CLB & Yes \\
\hline
\end{tabular}

$\mathrm{CBZ}=$ carbamazepine; $\mathrm{CLB}=$ clobazam; $\mathrm{CZP}=$ clonazepam; $\mathrm{DZP}=$ diazepam; $\mathrm{ESM}=$ ethosuximide; $\mathrm{GBP}=$ gabapentin; $\mathrm{KBr}=$ potassium bromide; $\mathrm{LEV}=$ levetiracetam; $\mathrm{LTG}=$ lamotrigine; $\mathrm{PB}=$ phenobarbital; $\mathrm{PER}=$ perampanel; $\mathrm{PHT}=$ phenytoin; RUF = rufinamide; TPM = topiramate; VPA = valproate; ZNS = zonisamide.

* ILAE classes $1-3$.

† ILAE classes 4-6.

The anterior insula is assumed to be involved in cognitive function, including attention, working memory, memory, language, and speech. ${ }^{11}$ The present evaluation of global IQ assessed all of these functions except memory and speech, and that of global DQ included language function. However, no significant postoperative change in global IQ/ DQ was detected after anterior insula resection. The same findings were obtained for verbal function. The left anterior insula may be crucial in speech production through articulation. ${ }^{24}$ Investigation of changes in attention, executive function, memory, language, and visuospatial skills before and after surgery showed that insular resection only affected oromotor speed, which may be related to speech production and articulation. ${ }^{17}$ Therefore, the test batteries we used might not have detected potential changes in articulation difficulties caused by insular resection. However, the present findings provide further evidence that the negative cognitive impact of insular resection on language function is insignificant in pediatric patients with insular epileptogenic lesions. Furthermore, attention and working memory may not change after this type of surgery.
Language and speech tasks show left-side dominance in activation of the anterior insula. ${ }^{15}$ However, left insular resection showed no significant effect, although only in 4 patients. Therefore, further studies are needed to determine whether left and right insular resection have different effects on cognitive function.

Seizures can result in cognitive impairment in children. ${ }^{25}$ Early age of seizure onset is also associated with poor cognitive function in children with FCD ${ }^{26}$ Furthermore, frequent IEDs can also affect cognition. ${ }^{27}$ Our pediatric patients with early seizure onset, frequent seizures, and widely distributed IEDs showed impaired cognition and development before surgery. Consequently, no further decline in postoperative cognitive function was found in this series. This finding may imply resection did not cause cognitive aggravation, and also that surgery prevented further cognitive decline. This proposal is supported by the experience of the 4 patients with preoperative developmental and cognitive regression or stagnation. Two of these patients with poor seizure outcome (cases 9 and 13) continued to decline until the last evaluation. In contrast, 
A

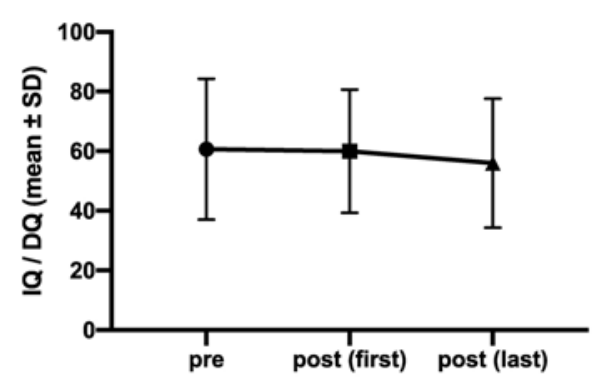

C

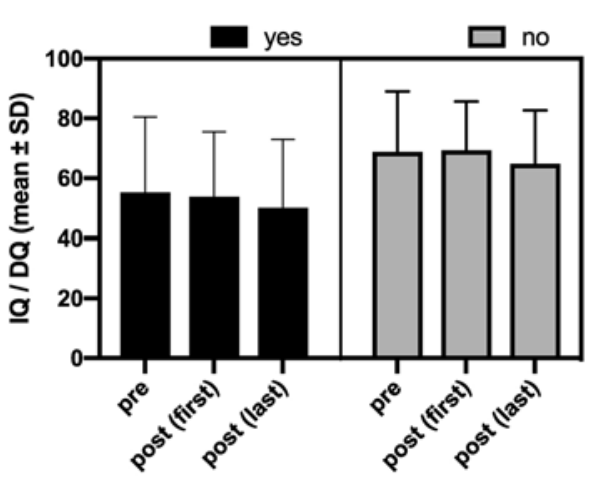

$\mathrm{E}$

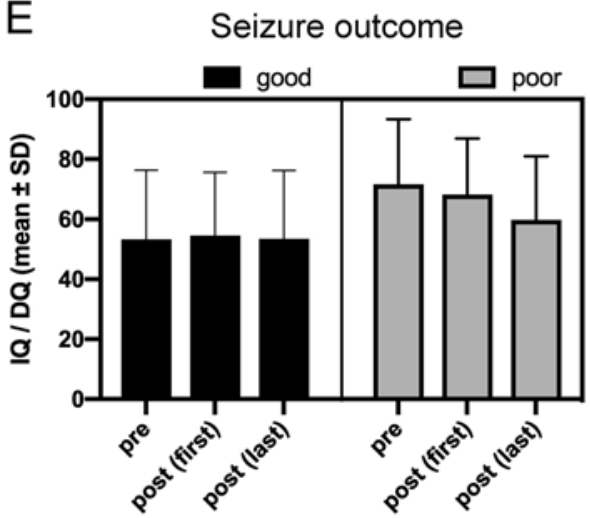

B

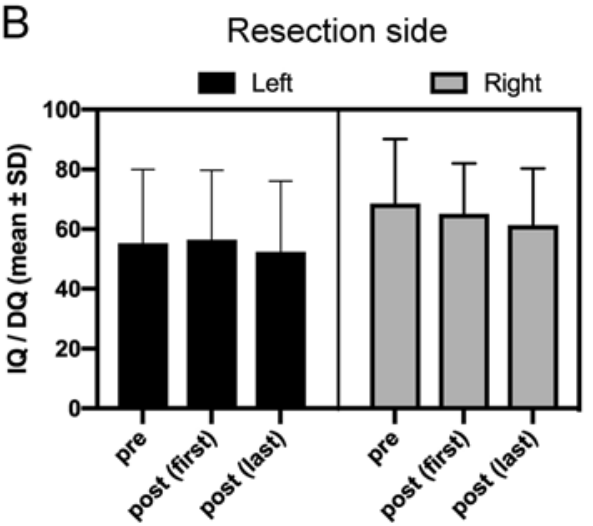

$\mathrm{D}$ AED reduction

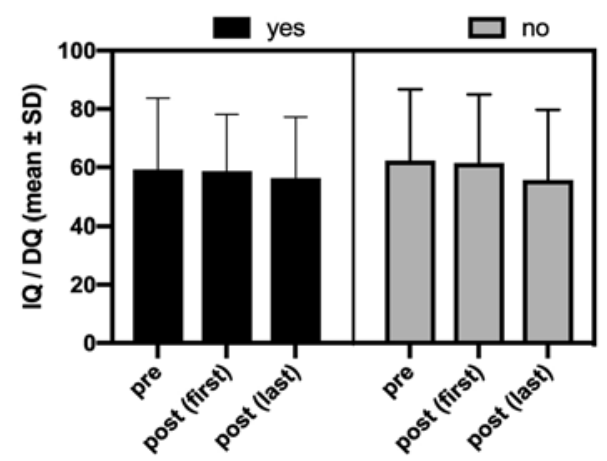

FIG. 2. Preoperative, first (within 4 months after surgery), and last (12 months or more after surgery) postoperative IQ/DQ scores. No significant changes were found in global score ( $\mathbf{A})$. Resection side (left/right), anterior insular resection (yes/no), AED reduction (yes/no), and seizure outcome (good/poor) did not significantly affect changes in scores between subjects (B-E).

the other 2 patients with seizure freedom (case 3) and worthwhile improvement (case 10) experienced no aggravation of cognition. This finding may indicate that cognitive aggravation can be prevented by reduction of seizures. Our finding of different cognitive outcomes despite the same seizure outcome could be because cognitive function is affected by both seizures and other factors, including IEDs. Long-term observation of cognitive function is necessary, and additional treatment may be required for patients with poor seizure outcome to improve the cognitive and developmental outcome.

Reduction in AED administration has no significant positive effect on cognitive function in the short- to midterm, although improvement can be expected in children. ${ }^{23}$ Possibly, many patients are still taking multiple AEDs even after reduction in the number or dosage of medications. Further investigation is necessary to confirm the benefit of fewer drugs.

This study was not intended to assess postoperative neurological complications, but 2 of our 5 patients who underwent posterior insular resection but no CST resection developed permanent hemiparesis, indicating that unexpected motor complications are not uncommon after posterior insular resection. This finding suggests that re- 

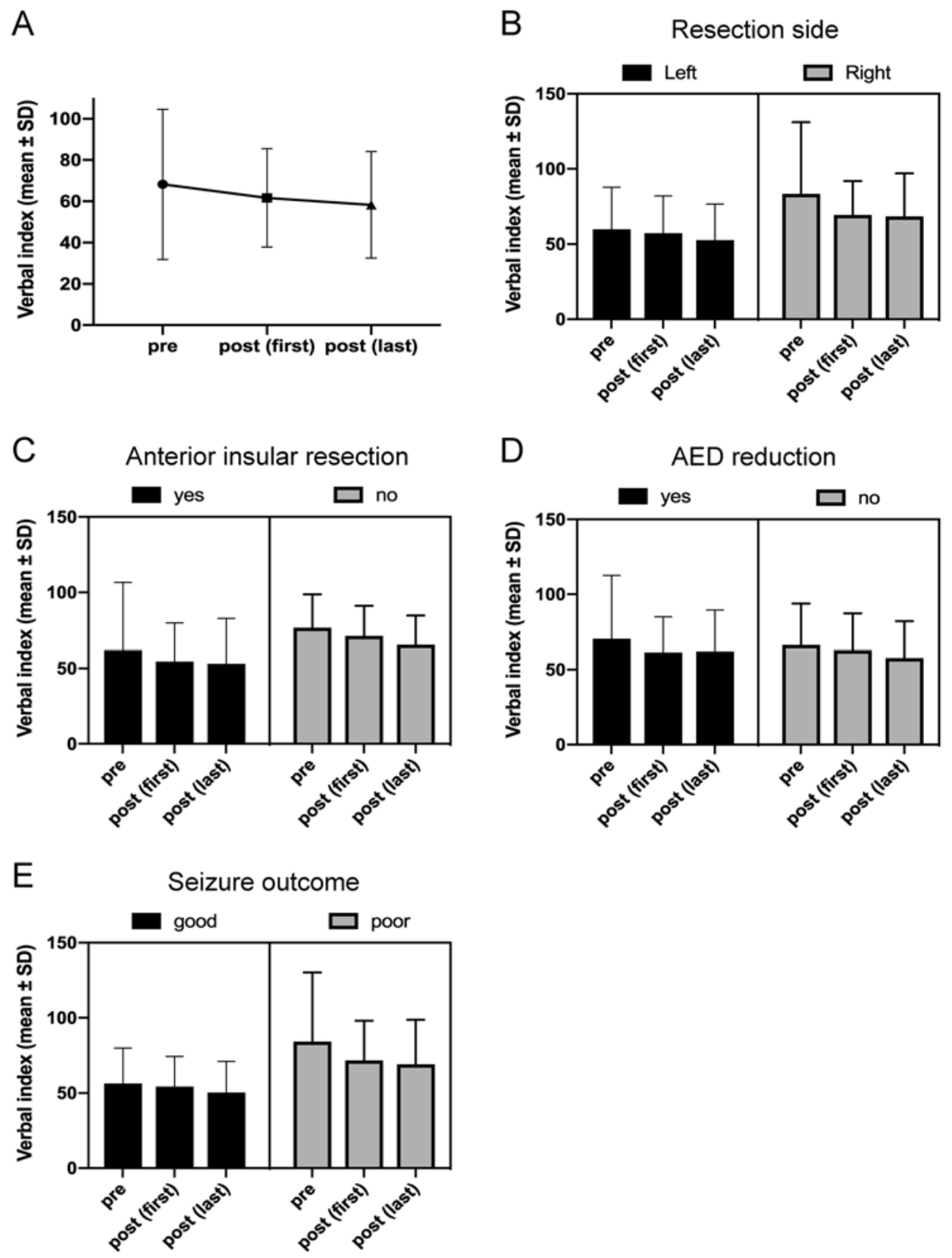

FIG. 3. Preoperative, first (within 4 months after surgery), and last (12 months or more after surgery) postoperative verbal index score. DQ scores are expressed as (language comprehension + language expression)/2. Verbal comprehension in the WISC was used as the index for IQ. No statistically significant changes were found in any scores (A, global verbal index score; B-E, between-subject factors).

section should be limited to avoid the risk of hemiparalysis, which is a relatively serious complication. In contrast, cognitive function and development should be prioritized in the presence of epileptic encephalopathy so the risk of motor paralysis after surgery may be acceptable.

This study has several limitations. First, evaluations of neuropsychological and developmental tests were combined to assess cognition. DQ consists of both cognitive function and motor function. Second, memory and speech that might be affected by insular resections were not evaluated. However, these functions may be difficult to accu- rately evaluate in children. Finally, this study investigated a small and heterogeneous cohort, including patients with severe intellectual and developmental disability. The floor effect makes changes in IQ difficult to detect in patients with severe intellectual disability. ${ }^{28,29}$ The floor effect is not well known in the DQ used in this study, but might complicate our results.

Despite these limitations, we did not observe any evidence for significant reduction in DQ/IQ after resection of the insula in our series, including in patients with intellectual and developmental disabilities who are often indi- 
cated for epilepsy surgery. These findings may be helpful in considering treatment strategies for the insular region.

\section{Conclusions}

Cognitive and developmental functions were not affected by epilepsy surgery involving the insula in children with intellectual and developmental disabilities. Insular cortex resection can be performed without significant reduction in $\mathrm{DQ} / \mathrm{IQ}$, but carries the risk of postoperative motor deficits.

\section{Acknowledgments}

We thank Satsuki Tabata for her technical support. This study was supported in part by an Intramural Research Grant (no. 28-4, Clinical Research for Diagnostic and Therapeutic Innovations in Developmental Disorders) for Neurological and Psychiatric Disorders of the NCNP.

\section{References}

1. Bouthillier A, Nguyen DK. Epilepsy surgeries requiring an operculoinsular cortectomy: operative technique and results. Neurosurgery. 2017;81(4):602-612.

2. Freri E, Matricardi S, Gozzo F, et al. Perisylvian, including insular, childhood epilepsy: Presurgical workup and surgical outcome. Epilepsia. 2017;58(8):1360-1369.

3. Gras-Combe G, Minotti L, Hoffmann D, et al. Surgery for nontumoral insular epilepsy explored by stereoelectroencephalography. Neurosurgery. 2016;79(4):578-588.

4. Jobst BC, Gonzalez-Martinez J, Isnard J, et al. The insula and its epilepsies. Epilepsy Curr. 2019;19(1):11-21.

5. Laoprasert P, Ojemann JG, Handler MH. Insular epilepsy surgery. Epilepsia. 2017;58(suppl 1):35-45.

6. Park YS, Lee YH, Shim KW, et al. Insular epilepsy surgery under neuronavigation guidance using depth electrode. Childs Nerv Syst. 2009;25(5):591-597.

7. von Lehe M, Parpaley Y. Insular cortex surgery for the treatment of refractory epilepsy. J Clin Neurophysiol. 2017;34(4): 333-339.

8. von Lehe M, Wellmer J, Urbach H, et al. Insular lesionectomy for refractory epilepsy: management and outcome. Brain. 2009;132(Pt 4):1048-1056.

9. Weil AG, Le NM, Jayakar P, et al. Medically resistant pediatric insular-opercular/perisylvian epilepsy. Part 2: Outcome following resective surgery. J Neurosurg Pediatr. 2016;18(5): 523-535.

10. Ikegaya N, Takahashi A, Kaido T, et al. Surgical strategy to avoid ischemic complications of the pyramidal tract in resective epilepsy surgery of the insula: technical case report. $J$ Neurosurg. 2018;128(4):1173-1177.

11. Kurth F, Zilles K, Fox PT, et al. A link between the systems: functional differentiation and integration within the human insula revealed by meta-analysis. Brain Struct Funct. 2010; 214(5-6):519-534.

12. Nguyen DK, Nguyen DB, Malak R, et al. Revisiting the role of the insula in refractory partial epilepsy. Epilepsia. 2009; 50(3):510-520.

13. Afif A, Minotti L, Kahane P, Hoffmann D. Anatomofunctional organization of the insular cortex: a study using intracerebral electrical stimulation in epileptic patients. Epilepsia. 2010;51(11):2305-2315.

14. Gasquoine PG. Contributions of the insula to cognition and emotion. Neuropsychol Rev. 2014;24(2):77-87.

15. Oh A, Duerden EG, Pang EW. The role of the insula in speech and language processing. Brain Lang. 2014;135: 96-103.
16. Ibañez A, Gleichgerrcht E, Manes F. Clinical effects of insular damage in humans. Brain Struct Funct. 2010;214(5-6): 397-410.

17. Boucher O, Rouleau I, Escudier F, et al. Neuropsychological performance before and after partial or complete insulectomy in patients with epilepsy. Epilepsy Behav. 2015;43:53-60.

18. Mullatti N, Landre E, Mellerio C, et al. Stereotactic thermocoagulation for insular epilepsy: lessons from successes and failures. Epilepsia. 2019;60(8):1565-1579.

19. Nickels KC, Zaccariello MJ, Hamiwka LD, Wirrell EC. Cognitive and neurodevelopmental comorbidities in paediatric epilepsy. Nat Rev Neurol. 2016;12(8):465-476.

20. Janszky J, Ebner A, Kruse B, et al. Functional organization of the brain with malformations of cortical development. Ann Neurol. 2003;53(6):759-767.

21. Fisher RS, Cross JH, French JA, et al. Operational classification of seizure types by the International League Against Epilepsy: position paper of the ILAE Commission for Classification and Terminology. Epilepsia. 2017;58(4):522-530.

22. Honda R, Kaido T, Sugai K, et al. Long-term developmental outcome after early hemispherotomy for hemimegalencephaly in infants with epileptic encephalopathy. Epilepsy Behav. 2013;29(1):30-35.

23. Boshuisen K, van Schooneveld MM, Uiterwaal CS, et al. Intelligence quotient improves after antiepileptic drug withdrawal following pediatric epilepsy surgery. Ann Neurol. 2015;78(1):104-114.

24. Baldo JV, Wilkins DP, Ogar J, et al. Role of the precentral gyrus of the insula in complex articulation. Cortex. 2011; 47(7):800-807.

25. Holmes GL. What is more harmful, seizures or epileptic EEG abnormalities? Is there any clinical data? Epileptic Disord. 2014;16(Spec No 1):S12-S22.

26. Korman B, Krsek P, Duchowny M, et al. Early seizure onset and dysplastic lesion extent independently disrupt cognitive networks. Neurology. 2013;81(8):745-751.

27. Nicolai J, Ebus S, Biemans DP, et al. The cognitive effects of interictal epileptiform EEG discharges and short nonconvulsive epileptic seizures. Epilepsia. 2012;53(6):1051-1059.

28. Whitaker S, Gordon S. Floor effects on the WISC-IV. Int J Dev Disabil. 2012;58(2):111-119.

29. Whitaker S, Wood C. The distribution of scaled scores and possible floor effects on the WISC-III and WAIS-III. $J$ Appl Res Intellect Disabil. 2007;21(2):136-141.

\section{Disclosures}

The authors report no conflict of interest concerning the materials or methods used in this study or the findings specified in this paper.

\section{Author Contributions}

Conception and design: Iwasaki, Ikegaya. Acquisition of data: Ikegaya, Kaneko, Kaido, Kimura, Sumitomo, Takahashi. Analysis and interpretation of data: Iwasaki, Ikegaya. Drafting the article: Ikegaya. Critically revising the article: Iwasaki, Ikegaya, Yamamoto. Reviewed submitted version of manuscript: Iwasaki, Ikegaya, Kaido, Kimura, Yamamoto, Sumitomo, Saito, Nakagawa, Sugai, Sasaki, Takahashi, Otsuki. Approved the final version of the manuscript on behalf of all authors: Iwasaki. Study supervision: Iwasaki, Kaneko, Yamamoto, Saito, Nakagawa, Sugai, Sasaki, Otsuki.

\section{Correspondence}

Masaki Iwasaki: National Center Hospital, National Center of Neurology and Psychiatry, Tokyo, Japan. iwa@ncnp.go.jp. 